Chem. Ber. 119, 3059-3066 (1986)

\title{
Bicyclo[4.1.1]octa-2,4-dien, -oct-2-en, -oct-3-en und -octan aus Norpinen
}

\author{
Manfred Christl ${ }^{*}$, Clemens Herzog ${ }^{1)}$ und Rainer Nusiser
}

Institut für Organische Chemie der Universität Würzburg, Am Hubland, D-8700 Würzburg

\section{Eingegangen am 2. Mai 1986}

Uber einen neuen Syntheseweg für Bicyclo[4.1.1]octa-2,4-dien (1), -oct-3-en (3) und -octan (4) sowie die erstmalige Darstellung von Bicyclo[4.1.1]oct-2-en (2) wird berichtet. Als gemeinsame Vorstufe diente 2,3-Dibrombicyclo[4.1.1]oct-3-en (7), das aus Norpinen (5) durch Addition von Dibromcarben und thermische Umlagerung des entstandenen Tricyclus 6 bereitet wurde.

\section{Bicyclo[4.1.1]octa-2,4-diene, -oct-2-ene, -oct-3-ene, and -octane from Norpinene}

A new route for the synthesis of bicyclo[4.1.1]octa-2,4-diene (1), -oct-3-ene (3), and -octane (4) is described as well as the first preparation of bicyclo[4.1.1]oct-2-ene (2). As common precursor 2,3-dibromobicyclo[4.1.1] ]oct-3-ene (7) has been utilized, obtained from norpinene (5) by addition of dibromocarbene and subsequent thermal rearrangement of the tricyclic product 6 formed.

Bicyclo[4.1.1]octa-2,4-dien (1) wurde erstmals von Paquette et al..$^{2)}$ in 16 Stufen aus Pentaerythrit dargestellt. Die dabei als Zwischenprodukt dienende cis-1,3-Cyclobutandicarbonsäure erhält man neuerdings bei guter Ausbeute einfacher, nämlich in vier Schritten aus Cyclopentadien über Benzvalen ${ }^{3}$. trans,trans-2,4-Diphenyl-cis-1,3-cyclobutandicarbonsäure nutzte man anhand der gleichen Methodik zur Bereitung von exo,anti-7,8-Diphenylbicyclo[4.1.1] octa-2,4-dien ${ }^{4)}$ und durch oxidativen Abbau der Phenylgruppen in einem geeigneten Stadium zur Synthese von Tricyclo[5.5.0.028]dodeca-3,5,9,11-tetraen ${ }^{5}$. Borden et al. ${ }^{6}$ gelangten ausgehend von 2-(Trimethylsiloxy)-1,3-butadien in 11 Stufen $\mathrm{zu} 1$. Sie stellten zunächst den Trimethylsilyl-enolether des Norpinanons her, cyclopropanierten diesen und kamen zur Vier-Kohlenstoff-Brücke durch Ringerweiterung. Mit einer verwandten Reaktionsfolge hatte man schon zuvor Nopinon in deuterierte Derivate von 7,7-Dimethylbicyclo[4.1.1] ]octa-2,4-dien umgewandelt ${ }^{7}$. Letztere Verbindung war erstmals aus $\alpha$-Pinen erhalten worden ${ }^{8}$. Eine durch Licht ausgelöste intramolekulare $[2+2]$-Cycloaddition ist der Schlüsselschritt bei der Synthese eines tricyclischen Derivats von 1 durch Gleiter et al. ${ }^{9}$. Bicyclo[4.1.1] oct-2-en-7-on entsteht bei einer photochemischen Umlagerung ${ }^{10 a)}$ und wurde zur Darstellung von Bicyclo[4.1.1] octa-2,4-dien-7-on verwendet ${ }^{106)}$.

Als erstes Derivat von 1 war wohl die 3-Brom-2,7,7-trimethyl-Verbindung durch Addition von Dibromcarben an $\alpha$-Pinen und thermolytische Ringerweiterung des gebildeten Tricyclus aufgebaut worden ${ }^{11}$. Mit Hilfe dieses Verfahrens hatten wir exo,syn-7,8-Dibrombicyclo[4.1.1] octa-2,4-dien, die Vorstufe für Octavalen, gewonnen ${ }^{12}$. Wir berichten jetzt über

(C) VCH Verlagsgesellschaft mbH, D-6940 Weinheim, 1986

$0009-2940 / 86 / 1010-3059 \$ 02.50 / 0$ 
die Anwendung dieses Verfahrens auf die Darstellung von 1 und seinen Hydroderivaten 2-4, was die Leistungsfähigkeit unserer Synthese von Norpinen (5) ${ }^{13)}$ unter Beweis stellt.

Hauptmotiv für diese Studie war ein besonderer Hochfeldeffekt im ${ }^{13} \mathrm{C}$-NMR-Spektrum eines 7,8-überbrückten 1-Derivats, den Sander und Gleiter ${ }^{14)}$ am Homoallylsignal der in der Briefumschlag-Konformation fixierten 1,3-Cycioheptadien-Einheit beobachtet hatten. Obwohl die ${ }^{13} \mathrm{C}$-chemischen Verschiebungen von 1, 2 und 4 schon bekannt waren ${ }^{2)}$, erwies sich die erneute Darstellung als notwendig, da wegen des Fehiens off-resonance-entkoppelter und protonengekoppelter Spektren die spezifische Zuordnung der Absorptionen von C-1,6 und $\mathrm{C}-7,8$ noch ausstand.

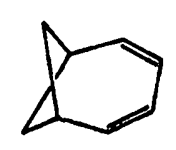

1

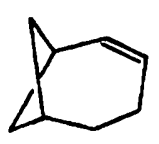

2

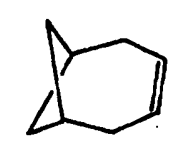

3<smiles>C1CC2CCC(C1)C2</smiles>

4

Norpinen (5) ergab mit Dibromcarben das Dibromtricyclooctan 6 mit $51 \%$ Ausbeute. In Acetonitril ging 6 bei $80^{\circ} \mathrm{C}$ in das Dibrombicycloocten 7 über (89\%). Das denkbare Isomere von 7 mit umgelagerter Dibromallyl-Einheit wurde nicht beobachtet. In Anlehnung an bekannte Reduktionen von Dichlorallylgruppen ${ }^{15,16 a)}$ behandelten wir 7 mit Natrium/tert-Butylalkohol und erhielten das gewünschte<smiles>CC(C)OC1CC2C=CC(C2)C1</smiles>

5<smiles>BrC1C2CC3CC1C3C2Br</smiles>

6<smiles>[CH]C1C2C=CC=CC1C2C</smiles>

1. $\left(\mathrm{CH}_{3}\right)_{3} \mathrm{CLi}$

2. $\mathrm{H}_{2} \mathrm{O}$<smiles>BrC1=CC2CC2C=C1</smiles>

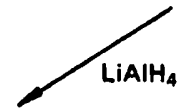<smiles>CC(C)(C)OC1=CC2CC2CC1</smiles>

9<smiles>C1CC2CC2CC2CC12</smiles>

2<smiles>[14CH2]C1CCCC2CC2C1</smiles>

4

Chem. Ber. 119 (1986) 


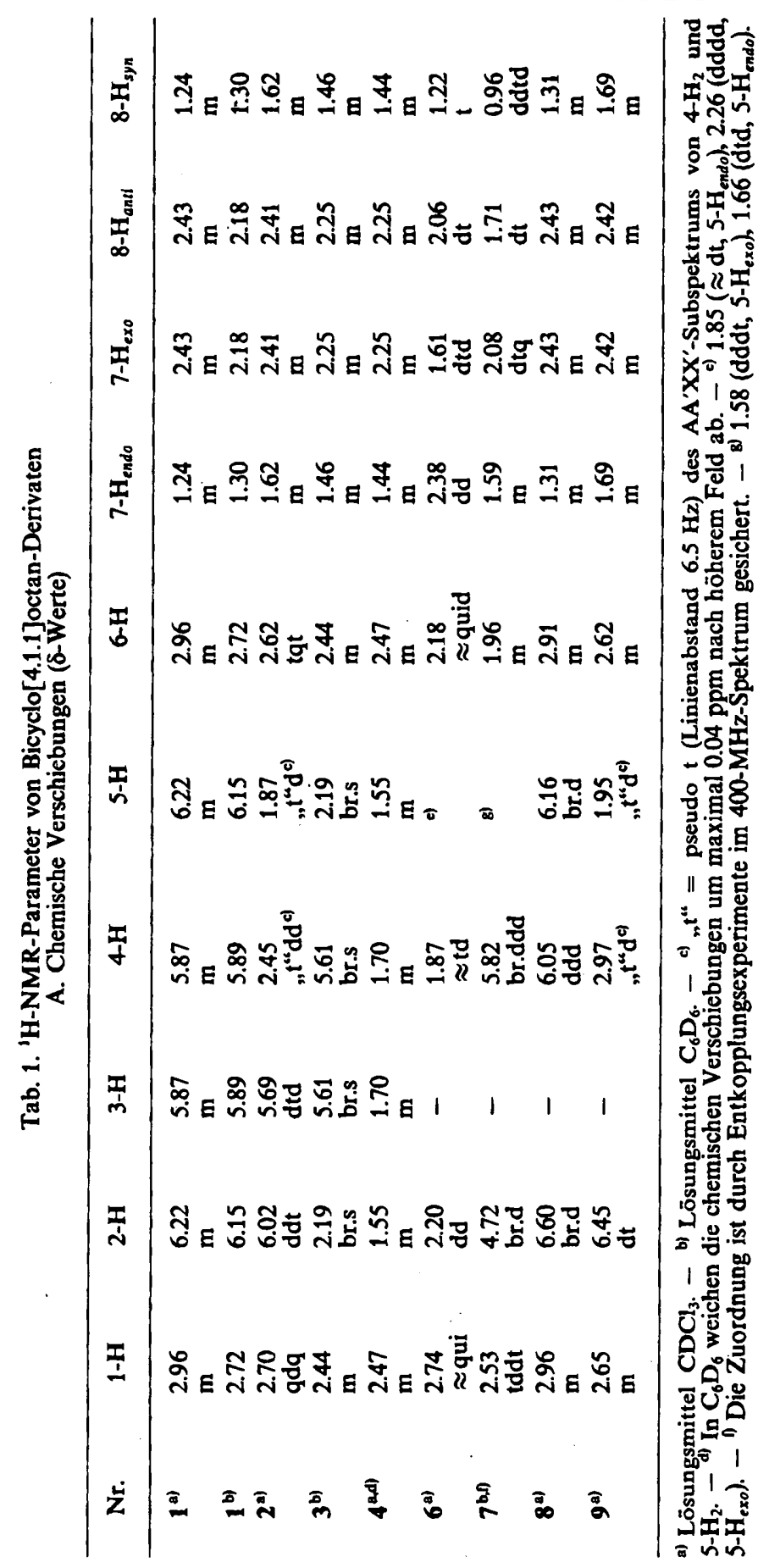

Chem. Ber. 119 (1986) 


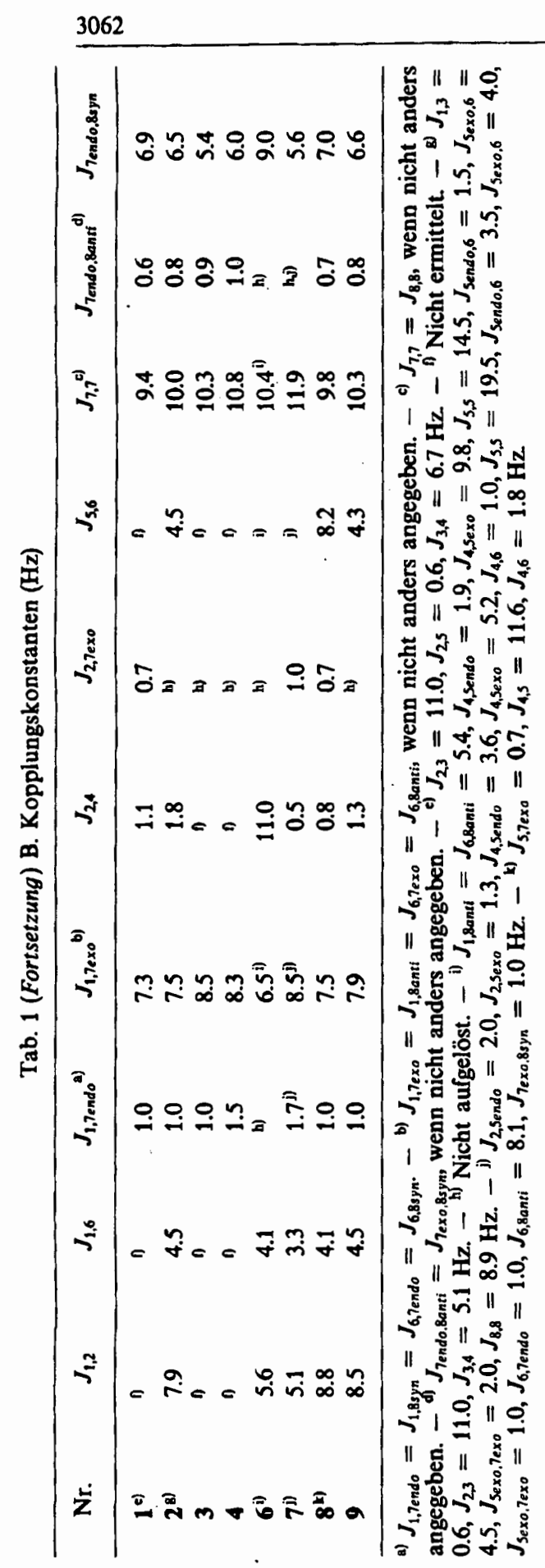

M. Christl, C. Herzog und R. Nusser

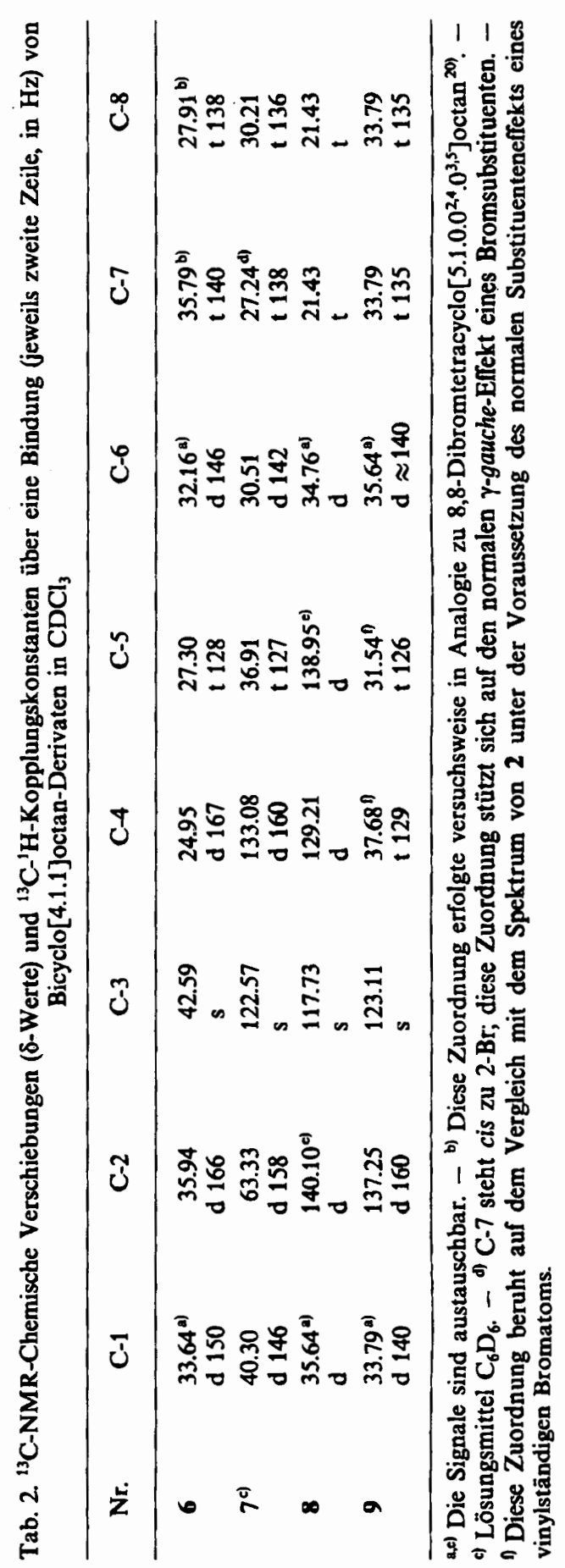

Chem. Ber. 119 (1986) 
3, wenn auch nur mit 3\% Ausbeute. Da die Substanzmenge für die NMR-Spektroskopie ausreichte, verzichteten wir auf die Erschließung eines ergiebigeren Wegs. Möglichkeiten dafür entnehme man der Arbeit mit der Synthese von Tricyclo[5.1.0.0 $\left.{ }^{28}\right]$ oct-4-en ${ }^{16 b)}$.

Die Umwandlungen von 7 in 1, 2 und 4 haben bewährte Reaktionsfolgen zum Vorbild ${ }^{12,16)}$. So eliminierten wir Bromwasserstoff aus 7 mit Hilfe von 1,8Diazabicyclo[5.4.0]undec-7-en (DBU) und kamen mit 53\% Ausbeute zu 3Brombicyclo[4.1.1] ]octa-2,4-dien (8), aus dem durch aufeinanderfolgende Einwirkung von tert-Butyllithium und Wasser bei $-78^{\circ} \mathrm{C}$ der Kohlenwasserstoff 1 (26\%) hervorging. Die Reduktion von 7 mit $\mathrm{LiAlH}_{4}$ erbrachte unter Allylumlagerung die Monobromverbindung $9(69 \%)$, die analog $z \mathrm{u} 8 \rightarrow 1$ in unsubstituiertes Bicyclo[4.1.1]oct-2-en (2) übergeführt wurde (43\%). Den gesättigten Kohlenwasserstoff 4 erhielten wir aus 2 durch katalytische Hydrierung (64\%).

Unser Weg zu 1 beginnt mit Cyclopentadien, umfaßt zehn Stufen und liefert eine Gesamtausbeute von ca. $1 \%$. Er ist damit erheblich kürzer und ergiebiger als der von Paquette et al. ${ }^{2}$, erreicht jedoch die Effizienz desjenigen von Borden et al. ${ }^{6 y}$ (elf Stufen, 3.9\%) nicht. Allerdings wurden die beiden letzten Schritte unserer Sequenz nur einmal ausgeführt, weshalb die zugehörigen Ausbeuten (53\%, $26 \%$ ) verbesserungsfähig sein sqllten. Der Vorteil der vorgestellten Route über Norpinen besteht darin, daß 1-4 aus einer gemeinsamen Vorstufe, nämlich 7, erhältlich sind.

Die Identităt von 1, 3 und 4 folgt aus dem Vergleich der NMR-Spektren mit den publizierten Daten ${ }^{2}$. Auch die Strukturen der neuen Verbindungen 2 und $6-9$ leiten sich zweifelsfrei aus den ${ }^{1} \mathrm{H}$ - (Tab. 1) und ${ }^{13} \mathrm{C}$-NMR-Spektren (Tab. 2) ab.

Da 7 nicht symmetrisch ist, wurden seine $400-\mathrm{MHz}^{-} \mathrm{H}-\mathrm{NMR}$-Signale nach erster Ordnung interpretiert. Dagegen verursachen 7-, 8- $\mathrm{H}_{2}$ von $1-4$ sowie 8 und $9 \mathrm{AA}^{\prime} \mathrm{XX}^{\prime}$-Spektren mit weiteren Aufspaltungen, die von 1-, 6-H und zum Teil auch von 2-, 5-H stammen. Die Kopplungskonstanten (Tab. 1B) wurden daher durch Analyse der AA'XX'-Spektren ${ }^{17}$ ) ermittelt; $J_{7,7}, J_{7 \text { endo, \&enti }}$ und $J_{\text {7endo,8syn }}$ haben das gleiche, wahrscheinlich das negative Vorzeichen. Beim Vergleich mit Norpinenen ${ }^{13}$ fällt auf, daß die geminale Kopplung $J_{\eta,}$ von $8.1-9.5$ auf $9.4-11.9 \mathrm{~Hz}$ vergrößert und die Fernkopplung zwischen den pseudoäquatorialen Protonen am Vierring $\left(J_{6 \text { ende.7syn }}\right.$ bzw. $\left.J_{\text {Tendo.8syn }}\right)$ von $8.0-8.6$ auf $5.4-7.0 \mathrm{~Hz}$ verkleinert ist. Anders als die entsprechenden Werte bei den Norpinenen und beim Norpinan-Derivat 6 sind die Fernkopplung $J_{\text {tendo.8ant }}(0.6-1.0 \mathrm{~Hz})$ und die vicinale Kopplung von $7-\mathrm{H}_{\text {endeo }}$ 8- $\mathrm{H}_{s y n}$ mit 1-, 6-H $(1.0-1.7 \mathrm{~Hz})$ aufgelöst. Zur Ermittlung der Wechselwirkungen zwischen 2-H, 3-H, 4-H und 5-H von 1 (Tab. 1B) wurde das 1-, 6-H-Signal entkoppelt und das resultierende $\mathrm{AA}^{\prime} \mathbf{X X}^{\prime}$-Spektrum analysiert ${ }^{1}{ }^{\prime} ; J_{2,3}, J_{2,4}, J_{25}$ und $J_{3,4}$ haben das gleiche, wahrscheinlich das positive Vorzeichen. Die Zuordnung der Signale von 7 stūtzt sich auf Entkopplungsexperimente, wobei sich die für Homoallylkopplungen $\left(J_{2,5}\right)$ bekannte Relation $J_{\text {trans }}>J_{c(s)}{ }^{18)}$ ergab.

Bezüglich der chemischen Verschiebungen von 7, 8- $\mathrm{H}_{2}$ weisen die Diene 1 und 8 die größte Differenz zwischen den Signalen auf, was hauptsächlich auf den Hochfeldwert von 7- $\mathrm{H}_{\text {endoo }}$ 8- $\mathrm{H}_{s y n}$ zurückzuführen ist. Dieses Phänomen hat möglicherweise die gleiche Ursache wie der besondere Hochfeldeffekt, der sich in den ${ }^{13} \mathrm{C}$-NMR-Signalen von C-7,8 in 1 und 8 manifestiert, und der in der nachstehenden Arbeit ${ }^{19}$ diskutiert wird. Dort sind auch die ${ }^{13} \mathrm{C}$ -

Chem. Ber. 119 (1986) 
NMR-Daten von 1-4 zusammengestellt, während Tab. 2 die Daten der bromsubstituierten Verbindungen 6-9 enthält.

Wir danken der Deutschen Forschungsgemeinschaft für eine Sachbeihilfe.

\section{Experimenteller Teil}

Geräte: Lit. ${ }^{\text {(2b). }}$

3,3-Dibromtricyclo[4.1.1.0 ${ }^{2,4}$ Joctan (6): Unter Stickstoff wurden $1.83 \mathrm{~g}(19.4 \mathrm{mmol}) 5^{13)}$ in $51.0 \mathrm{~g}$ Petroleumbenzin $\left(60-70^{\circ} \mathrm{C}\right)$ bei $-15^{\circ} \mathrm{C}$ mit $8.77 \mathrm{~g}(78.3 \mathrm{mmol})$ Kalium-tert-butoxid versetzt. Unter Rühren tropfte man bei -15 bis $-10^{\circ} \mathrm{C} 10.1 \mathrm{~g}(3.58 \mathrm{ml}, 39.9 \mathrm{mmol})$ Bromoform während 10 min zu, ließ dann im Eisbad auftauen $(1 \mathrm{~h})$, hydrolysierte, trennte die Phasen, extrahierte die wäßrige Phase dreimal mit je $20 \mathrm{ml}$ Ether, trocknete die vereinigten organischen Phasen über $\mathrm{Na}_{2} \mathrm{SO}_{4}$ und engte i. Vak. ein. Aus dem Rückstand destillierten bei $42-45^{\circ} \mathrm{C} / 0.02$ Torr $2.66 \mathrm{~g}(51 \%) 6$ als farblose Flüssigkeit. - MS $(70 \mathrm{eV}): \mathrm{m} / \mathrm{z}=268$, 266, 264 (1.3, 2.6, 1.3\%, M+), 105 (99), 79 (100). - NMR: Tab. 1, 2.

2,3-Dibrombicyclo[4.1.1 Joct-3-en (7): $3.98 \mathrm{~g}$ (15.0 mmol) 6 wurden in $25 \mathrm{ml}$ Acetonitril $3 \mathrm{~d}$ auf $80^{\circ} \mathrm{C}$ erhitzt. Nach Einengen i. Vak. destillierten aus dem Rückstand bei $51-53^{\circ} \mathrm{C} /$ $10^{-3}$ Torr $3.54 \mathrm{~g}(89 \%) 7$ als farblose Flüssigkeit. - IR (Film): $1633 \mathrm{~cm}^{-1}(\mathrm{C}=\mathrm{C}$, schwach). - MS (70 eV): $m / z=268,266,264\left(6,12,6 \%, M^{+}\right), 105(100), 79(90), 77$ (90). NMR: Tab. 1, 2.

$$
\begin{array}{llllllll}
\mathrm{C}_{8} \mathrm{H}_{10} \mathrm{Br}_{2} \text { (266.0) Ber. C } 36.12 \text { H } 3.79 \text { 6: Gef. C } 36.35 \text { H } 3.70 \\
& & & \text { 7: Gef. C } 36.09 \text { H } 3.77
\end{array}
$$

3-Brombicyclo[4.1.1]octa-2,4-dien (8): Man ließ die Lösung von $1.30 \mathrm{~g}$ (4.89 mmol) 7 und $2.97 \mathrm{~g}$ (19.5 mmol) 1,8-Diazabicyclo[5.4.0] undec-7-en (DBU) in $10 \mathrm{ml}$ Benzol $5 \mathrm{~d}$ bei $22^{\circ} \mathrm{C}$ stehen, versetzte dann mit $20 \mathrm{ml}$ Wasser, trennte die Phasen und extrahierte die wäßrige Phase zweimal mit je $20 \mathrm{ml}$ Benzol. Die vereinigten Benzolphasen wurden zweimal mit je $20 \mathrm{ml} 2 \mathrm{~N} \mathrm{HCl}$ gewaschen, mit gesättigter $\mathrm{NaHCO}_{3}$-Lösung neutralisiert und mit $\mathrm{Na}_{2} \mathrm{SO}_{4}$ getrocknet. Nach Einengen i. Vak. destillierten bei $60-65^{\circ} \mathrm{C}(\mathrm{Bad}) / 2$ Torr $480 \mathrm{mg}(53 \%) 8$ als farbloses Ol aus dem Rückstand. - IR (Film): $1613 \mathrm{~cm}^{-1}(\mathrm{C}=\mathrm{C})$. - MS (70 eV): $m / z=186,184$ (13, 13\%, M+), 105 (100), 79 (46), 77 (56). - NMR: Tab. 1, 2.

$$
\mathrm{C}_{8} \mathrm{H}_{9} \mathrm{Br}(185.1) \text { Ber. C } 51.92 \text { H } 4.90 \text { Gef. C } 51.85 \text { H } 4.78
$$

Bicyclo[4.1.1]octa-2,4-dien (1): Unter Stickstoff und Rühren tropfte man zu $353 \mathrm{mg}$ (1.91 $\mathrm{mmol}) 8$ in $5 \mathrm{ml}$ absol. Ether bei $-78^{\circ} \mathrm{C}$ innerhalb von $15 \mathrm{~min} 2.86 \mathrm{mmol}(2.29 \mathrm{ml}, 1.25 \mathrm{~N}$ in Pentan) tert-Butyllithium. Es entstand eine rote Lösung. Man ließ $1 \mathrm{~h}$ weiterrühren, gab bei $-78^{\circ} \mathrm{C}$ Wasser zu, bis die Rotfärbung verschwand, ließ auftauen, trennte die Phasen, extrahierte die wäßrige Phase zweimal mit je $5 \mathrm{ml}$ Ether, trocknete die vereinigten Etherphasen mit $\mathrm{Na}_{2} \mathrm{SO}_{4}$ und destilliette den Ether über eine 20-cm-Vigreuxkolonne (Bad bis $50^{\circ} \mathrm{C}$ ) ab. Flüchtige Bestandteile des Rückstands wurden bei $10^{-2}$ Torr verdampft und in einer auf $-78^{\circ} \mathrm{C}$ gekühlten Vorlage kondensiert. Mit Hilfe der präparativen Gaschromatographie (Säule $6 \mathrm{~m}, 5 \% \mathrm{NaOH}$ und $20 \%$ Carbowax $20 \mathrm{M}$ auf Chromosorb $\mathrm{P}, 90^{\circ} \mathrm{C}$ ) isolierte man aus dem Kondensat $52.0 \mathrm{mg}(26 \%) 1$ als farblose Flüssigkeit. Die NMRSpektren stimmen mit den Literaturangaben ${ }^{2)}$ überein. - ${ }^{1}$ H-NMR: Tab. 1. - ${ }^{13}$ C-NMR: Lit. ${ }^{19)}$.

Bicyclo[ 4.1.1 Joct-3-en (3): Unter Rühren tropfte man innerhalb von $30 \mathrm{~min}$ die Lösung von $1.90 \mathrm{~g}$ (7.14 mmol) 7 und $2.12 \mathrm{~g}(28.6 \mathrm{mmol})$ tert-Butylalkohol in $80 \mathrm{ml}$ Ether $\mathrm{zu} 656 \mathrm{mg}$

Chem. Ber. 119 (1986) 
(28.6 mmol) Natrium in $100 \mathrm{ml}$ siedendem Ether. Danach erhitzte man 3 d unter Rúckfluß, hydrolysierte, trennte die Phasen, extrahierte die wäßrige Phase zweimal mit je $10 \mathrm{ml} \mathrm{n}$ Pentan, wusch die vereinigten organischen Phasen sechsmal mit je $50 \mathrm{ml}$ Wasser, trocknete sie mit $\mathrm{Na}_{2} \mathrm{SO}_{4}$, versetzte sie mit Natriumhydrid (ca. $4 \mathrm{~g}$, 80proz in Paraffinöl), bis keine Gasentwicklung mehr auftrat, destillierte das Solvens über eine 20-cm-Vigreuxkolonne (Bad bis $50^{\circ} \mathrm{C}$ ) ab, verdampfte die flüchtigen Anteile des Rückstands bei $10^{-2}$ Torr und kondensierte sie in einer auf $-78^{\circ} \mathrm{C}$ gekühiten Vorlage. Mit Hilfe der präparativen Gaschromatographie (Säule wie bei 1) isolierte man aus dem Kondensat $25 \mathrm{mg} \mathrm{(3 \% )} 3$ als farblose Flüssigkeit. Die NMR-Spektren stimmen mit den Literaturangaben ${ }^{2)}$ überein. ${ }^{1}$ H-NMR: Tab. 1. - ${ }^{13}$ C-NMR: Lit. ${ }^{19}$.

3-Brombicyclo[4.1.1 Joct-2-en (9): Man versetzte $1.30 \mathrm{~g}$ (4.89 mmol) 7 in $20 \mathrm{ml}$ Ether bei $20^{\circ} \mathrm{C}$ mit $280 \mathrm{mg}(7.36 \mathrm{mmol})$ Lithiumaluminiumhydrid und rührte $24 \mathrm{~h}$. Danach kochte man 3 h unter RückfluB, hydrolysierte unter Eiskühlung und versetzte mit $30 \mathrm{ml} 2 \mathrm{~N} \mathrm{HCl}$, wodurch sich zwei homogene Phasen bildeten. Man trennte diese, extrahierte die wäßrige Phase fünfmal mit je $5 \mathrm{ml}$ Ether, neutralisierte die vereinigten organischen Phasen mit gesättigter $\mathrm{NaHCO}_{3}$-Lösung, trocknete sie mit $\mathrm{Na}_{2} \mathrm{SO}_{4}$ und engte i. Vak. ein. Aus dem Rückstand destillierten bei $60-65^{\circ} \mathrm{C}(\mathrm{Bad}) / 2$ Torr $635 \mathrm{mg}(69 \%) 9$ als farblose Flüssigkeit. - IR (Film): $1640 \mathrm{~cm}^{-1}(\mathrm{C}=\mathrm{C})$. - MS $(70 \mathrm{eV}): m / z=188,186\left(9,10 \%, \mathrm{M}^{+}\right), 79$ (100), 66 (92), 65 (90); exakte Masse: Ber. 188.0021, Gef. 188.0020. - NMR: Tab. 1, 2.

$$
\mathrm{C}_{8} \mathrm{H}_{11} \mathrm{Br} \text { (187.1) Ber. C } 51.35 \text { H } 5.92 \text { Gef. C } 50.37 \text { H } 6.11
$$

Bicyclo[4.1.1 Joct-2-en (2): Aus $820 \mathrm{mg}$ (4.38 mmol) 9 erhielt man gemäß der für die Umwandlung $8 \rightarrow 1$ angegebenen Vorschrift (der Brom-Lithium-Austausch verursachte hier keine Rotfärbung) nach der präparativen Gaschromatographie $205 \mathrm{mg}(43 \%) 2$ als farblose Flüssigkeit. - IR (Film): $1648 \mathrm{~cm}^{-1}(\mathrm{C}=\mathrm{C})$. - MS (70 eV): $m / z=108\left(9 \%, \mathrm{M}^{+}\right), 93(38)$, 80 (41), 79 (74), $67(100), 66(48), 41$ (50), 39 (44); exakte Masse: Ber. 108.0938, Gef. 108.0937. 'H-NMR: Tab. 1. - ${ }^{13}$ C-NMR: Lit. ${ }^{19}$.

Bicyclo[4.1.1]octan (4): $165 \mathrm{mg}$ (1.53 mmol) 2 wurden in $20 \mathrm{ml}$ Ether in Gegenwart von $100 \mathrm{mg} \mathrm{Pd} /$ Aktivkohle $6 \mathrm{~h}$ unter Wasserstoff (1 bar) gerührt. Man filtrierte die Reaktionslösung durch Kieselgel $(5 \mathrm{~g}, 10 \mathrm{ml}$ Pentan als Laufmittel) und arbeitete dann wie bei 1 auf. Durch präparative Gaschromatographie erhielt man $107 \mathrm{mg}(64 \%) 4$ als farblosen, bei ca. $20^{\circ} \mathrm{C}$ schmelzenden Feststoff. Die NMR-Spektren stimmen mit den Literaturangaben ${ }^{2)}$ überein. - ${ }^{1}$ H-NMR: Tab. 1. - ${ }^{13}$ C-NMR: Lit. ${ }^{19}$.

\section{CAS-Registry-Nummern}

1: 61885-53-8 / 2: 16544-26-6 / 3: 61885-54-9 / 4: 7078-34-4 / 5: 7095-82-1 / 6: 103149 . 67-3 / 7: $103149-68-4$ / 8: $103149-69-5 / 9: 103149-70-8$

\footnotetext{
") Aus der Dissertation C. Herzog, Univ. Würzburg 1986.

${ }^{2)} R$. Gleiter, P. Bischof, W. E. Volz und $L$. A. Paquette, J. Am. Chem. Soc. 99, 8 (1977).

${ }^{3)} H$. Leininger, $F$. Lanzendörfer und M. Christl, Chem. Ber. 116, 669 (1983).

4) K. L. Chasey, L. A. Paquette und J. F. Blount, J. Org. Chem. 47, 5262 (1982).

5) L. A. Paquette, J. Dressel und K. L. Chasey, J. Am. Chem. Soc. 108, 512 (1986).

6) T.-K. Yin, J. G. Lee und W. T. Borden, J. Org. Chem. 50, 531 (1985)

" A. R. Browne, A. F. Drake, F. R. Kearney, S. F. Mason und L. A. Paquette, J. Am. Chem. Soc. 105, 6123 (1983).

${ }^{8}$ S. D. Young und W. T. Borden, Tetrahedron Lett. 1976, 4019.

9) R. Gleiter, W. Sander, H. Irngartinger und A. Lenz, Tetrahedron Lett. 23, 2647 (1982).
}

Chem. Ber. 119 (1986) 
10) 10a) W. F. Erman und H. C. Kretschmar, J. Am. Chem. Soc. 89, 3842 (1967); U. H. Brinker und L. König, Chem. Ber. 116, 894 (1983). - ${ }^{10 b}$ L. König und U. H. Brinker, Chem. Ber. 119,383 (1986).

11) J. Hatem und B. Waegell, Tetrahedron Lett. 1971, 2069; G. Mehta und S. C. Narang, Indian J. Chem. 10, 1057 (1972).

(2) 12a) M. Christl und $R$. Lang. J. Am. Chem. Soc. 104, 4494 (1982). - ${ }^{12 b)}$ M. Christl, R. Lang und C. Herzog, Tetrahedron 42,1585 (1986).

13) C. Herzog, R. Lang, D. Brückner, P. Kemmer und M. Christl. Chem. Ber. 119, 3027 (1986).

14) $W$. Sander und $R$. Gleiter, Chem. Ber. 118, 2548 (1985).

15) R. P. Johnson, A. Exarchou, C. W. Jefford und R. C. Hahn, J. Org. Chem. 42, 3758 (1977).

16) 16a) $M$. Christl, C. Herzog, D. Brückner und R. Lang. Chem. Ber. 119, 141 (1986). - ${ }^{66)} M$. Christl, C. Herzog und P. Kemmer, Chem. Ber. 119, 3045 (1986), voranstehend.

${ }^{17)} H$. Günther, NMR-Spektroskopie, 2. Aufl, Thieme, Stuttgart 1983.

18) $M$. Barfield und S. Sternhell, J. Am. Chem. Soc. 94, 1905 (1972).

19) M. Christl und C. Herzog, Chem. Ber. 119, 3067 (1986), nachstehend.

20) $M$. Christl und $W$. Buchner, Org. Magn. Reson. 11, 461 (1978). 